\title{
Percepciones de Investigadores en Formación respecto al Desarrollo de Competencias
}

\author{
Perceptions of Early Career Researchers Regarding Competence Development
}

Percepções de Pesquisadores em Formação respeito do Desenvolvimento de Competências

Lourdes Villardón-Gallego* https://orcid.org/0000-0001-7011-2444

Departamento de Didáctica y Desarrollo Curricular, Facultad de Psicología y Educación. Universidad de Deusto, Bilbao, España.

Beatriz Villarejo Carballido** http://orcid.org/0000-0002-0369-9115

Deusto Education Research-eDucaR, Facultad de Psicología y Educación. Universidad de Deusto, Bilbao, España

$\nabla$

Recibido: 08-05-18 Revisado: 14-07-18 Aceptado: 07-10-18 Publicado: 20-12-18

- Resumen. Los estudios de doctorado conducen al nivel más alto de cualificación académica, promoviendo el desarrollo de competencias que habiliten para el desempeño como líderes en la generación de conocimiento científico y en la aplicación del mismo a la mejora de la sociedad, a través de la investigación y la innovación. El proceso de adquisición de competencias supone un recorrido formativo exigente y de larga duración que conlleva dificultades que es necesario vencer con ayuda de factores tanto personales como contextuales. En esta investigación se ha recogido información sobre las percepciones de los y las investigadoras (8 participantes, 5 mujeres y 3 hombres) en formación con respecto al proceso de aprendizaje en los tres momentos del programa de doctorado: expectativas de aprendizaje, dificultades y recursos en el inicio, dificultades y apoyos experimentados, así como aprendizajes parciales percibidos en el proceso, y aprendizajes percibidos al final, una vez defendida y aprobada la tesis doctoral. Los resultados han permitido identificar los aspectos contextuales e individuales fundamentales en cada uno de los tres momentos de la formación de las personas investigadoras. Estos hallazgos tienen importantes implicaciones para la planificación y el desarrollo de la formación doctoral.
Palabras clave:

doctorado,

formación

investigadora, desarrollo de

competencias, afectividad en el aprendizaje, identidad investigadora. 
Abstract. Doctoral studies lead to the highest level of academic qualification, promoting the development of competences that enable individuals to perform as leaders in the generation of scientific knowledge and its application in the improvement of society, through research and innovation. The process of acquiring competences involves a demanding and long-lasting training path that entails difficulties that must be overcome with the help of both personal and contextual factors. In this research, information has been gathered on the perceptions of trainee researchers (8 participants: 5 women and 3 men) regarding the learning process at the three moments of the doctoral program: learning expectations, difficulties and resources at the beginning, difficulties and support experienced, as well as partial learning perceived in the process, and learning perceived at the end, once the doctoral thesis has been defended and approved. The results have made it possible to identify the fundamental contextual and individual aspects in each of the three moments of the researchers' training. These findings have important implications for the planning and development of doctoral training programs.

- Resumo. Os estudos de doutorado conduzem ao nível mais alto de qualificação acadêmica, promovendo o desenvolvimento de competências que facilitem o desempenho como líderes na geração de conhecimento científico e na aplicação deste conhecimento à melhora da sociedade, através da pesquisa e inovação. $O$ processo de aquisição de competências supõe um percurso formativo exigente e de longa duração, que acarreta dificuldades que é preciso vencer com ajuda de fatores pessoais e contextuais. Nesta pesquisa levantou-se informação sobre as percepções dos e das pesquisadoras (8 participantes, 5 mulheres e 3 homens) em formação a respeito do processo de aprendizagem nos três momentos do programa de doutorado: expectativas de aprendizagem, dificuldades e recursos no início, dificuldades e apoios experimentados, assim como a aprendizagem parcial percebida no processo e a aprendizagem percebida no fim, uma vez defendida e aprovada a tese doutoral. Os resultados permitiram identificar os aspectos contextuais e individuais fundamentais em cada um dos três momentos da formação dos pesquisadores. Estes achados têm importantes implicações para o planejamento e o desenvolvimento da formação doutoral.
Keywords:

doctoral training, research training, development of competences, affectivity in learning, researcher identity

Palavras-chave: doutorado, formação em pesquisa, desenvolvimento de competências, afetividade na aprendizagem, identidade pesquisadora. 


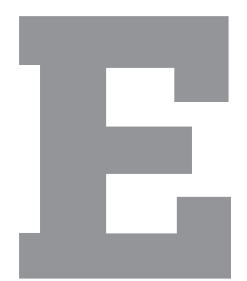

1 doctorado permite obtener un conocimiento avanzado, desarrollar funciones de liderazgo y realizar investigaciones (Gregor \& O’Brien, 2015; Hoyne, Alessandrini, \& Fellman, 2016). En los últimos años, el doctorado ha evolucionado acorde con las nuevas demandas que se imponen sobre los mismos (Melin \& Janson, 2006), las cuales requieren la enseñanza de nuevas habilidades y competencias. Estos aprendizajes se desarrollan a la vez que se va configurando la identidad profesional.

\section{Formación de investigadores basada en competencias}

En España las enseñanzas del doctorado están reguladas por el Real Decreto 99/2011 de 28 de enero. Entre los principios que fundamentan dicha normativa está la intención de que el doctorado tenga un papel protagonista en la intersección entre el Espacio Europeo de Educación Superior (EEES) y el Espacio Europeo de Investigación (EEI). Se considera que las personas doctoras deben ser protagonistas principales en la generación, transferencia y adecuación del I+D+i liderando el transvase desde el conocimiento hasta el bienestar de las sociedad. Por ello, es fundamental una formación excelente de las personas investigadoras. Sobre este perfil de egreso, considerando las funciones a desempeñar, se plantea desarrollar una serie de competencias.

ElMarco Español de Cualificaciones para la Educación Superior (MECES), regulado por el Real Decreto 1027/2011, de 15 de julio, en su Artículo 8, centra el nivel cuatro en la formación avanzada de técnicas de investigación. Tomando como base los aprendizajes señalados en el MECES, el Artículo 5 del Real Decreto 99/2011 incluye dos grupos de competencias que debe aprender el alumnado del doctorado. El primer grupo hace referencia a las competencias básicas (CB):

- CB1. Comprensión sistemática de un campo de estudio y dominio de las habilidades y métodos de investigación relacionados con dicho campo.

- CB2. Capacidad de concebir, diseñar o crear, poner en práctica y adoptar un proceso sustancial de investigación o creación.

- CB3. Capacidad para contribuir a la ampliación de las fronteras del conocimiento a través de una investigación original.

- CB5. Capacidad de realizar un análisis crítico y de evaluación y síntesis de ideas nuevas y complejas.

- CB6. Capacidad de comunicación con la comunidad académica y científica y con la sociedad en general acerca de sus ámbitos de conocimiento en los modos e idiomas de uso habitual en su comunidad científica internacional.

- CB7. Capacidad de fomentar, en contextos académicos y profesionales, el avance científico, tecnológico, social, artístico o cultural dentro de una sociedad basada en el conocimiento.

El segundo grupo incluye aquellas capacidades y destrezas que favorecen una alta capacitación profesional en ámbitos vinculados con la innovación (CA): 
- CA1. Desenvolverse en contextos en los que hay poca información específica.

- CA2. Encontrar las preguntas claves que hay que responder para resolver un problema complejo.

- CA3. Diseñar, crear, desarrollar y emprender proyectos novedosos e innovadores en su ámbito de conocimiento.

- CA4. Trabajar tanto en equipo como de manera autónoma en un contexto internacional o multidisciplinar.

- CA5. Integrar conocimientos, enfrentarse a la complejidad y formular juicios con información limitada.

- CA6. Crítica y defensa intelectual de soluciones.

Por su parte, Durette, Fournier y Lafon (2016) organizan los aprendizajes y competencias que se adquieren durante el doctorado en seis grupos:

a) Competencias de conocimiento y técnicas especializadas de un ámbito específico de conocimiento o técnicas concretas.

b) Competencias transversales "formalizables", que se utilizan en variedad de situaciones profesionales y pueden ser "aprendidas" en cursos, como idiomas, gestión por proyectos o comunicación.

c) Competencias transversales "no formalizables", que se utilizan en un rango importante de situaciones profesionales, pero no pueden ser "aprendidas" a través de cursos formales, comola capacidad de afrontar problemas complejos, capacidad para colaborar, liderazgo, innovación, visión global, etc.

d) Disposiciones, que incluyen aptitudes y cualidades como el rigor y la creatividad.

e) Comportamientos, que van desde el manejo del estrés a la perseverancia e incluyen, entre otros, la resiliencia, el dinamismo y la honestidad.

f) Metacompetencias, que constituyen aquellas competencias útiles para desarrollar las propias competencias o aplicarlas más eficazmente en situaciones profesionales. Son la capacidad para aprender y la capacidad de adaptación.

Cada programa de doctorado, normalmente con un alto grado de autonomía, debe poner los medios necesarios para favorecer el logro de las competencias mencionadas por parte del alumnado.

\section{Factores influyentes en la formación de investigadores}

En el desarrollo de estas competencias hay que tener en cuenta numerosos aspectos, tanto contextuales como personales, que interfieren facilitando o dificultando el aprendizaje (McAlpine \& Lucas, 2011). 
Lovitts (2008) investigó factores facilitadores y obstáculos en diferentes programas de doctorado de ciencias puras y ciencias sociales de dos universidades estadounidenses. Encontró que el alumnado que no completaba el doctorado tenía dificultades para encontrar ideas interesantes, poseía menos recursos personales y sociales, y su director o directora no realizaba un acompañamiento adecuado durante el proceso. Por su parte, Furr y Brown-Rice (2016) identificaron varios comportamientos en la supervisión de la tesis que obstaculizan el avance del estudiante, tales como la falta de honestidad, la tardanza excesiva en dar feedback, escasas habilidades para la supervisión y la dificultad para controlar las emociones.

Por otro lado, Lovitts (2008) identificó en el alumnado que culminó el doctorado una fuerte motivación por la investigación que estaba realizando, una asesoría adecuada e interacciones positivas tanto con su director o directora como con los y las compañeras. : Juniper, Walsh, Rochardson y Morley (2012) confirmaron la relación positiva entre el bienestar de las personas doctorandas y la existencia de relaciones sociales adecuadas.

Así lo visibiliza Ng (2017), quien analizó sus propias emociones mientras realizaba el doctorado y cómo éstas afectaban al proceso de elaboración de tesis. En la misma línea, Morrison-Saunders, Moore, Hughes y Newsome (2010) señalaron que el doctorado causa en el alumnado la sensación de estar en una auténtica "montaña rusa", ya que éste puede experimentar tanto emociones negativas como positivas. Anderson (2017) corroboró que el proceso doctoral afecta a los pensamientos, acciones, conductas y emociones del alumnado, aspectos que influyen, a su vez, en la toma de decisiones durante el proceso de aprendizaje con respecto a las demandas intelectuales exigidas (Cantwell, Scevak, Bourke, \& Hoolbrok, 2012; McLaughlin, 2003). Por ello, es importante el papel de las emociones en el aprendizaje (McLaughlin, 2003) y viceversa.

Diversas investigaciones destacan el papel fundamental del asesoramiento y del apoyo social en las emociones. Con respecto al asesoramiento, Pyhältö y Keskinen (2012) apuntaron que el papel de la persona supervisora es fundamental, entre otras aspectos, en la promoción e integración del alumnado en la comunidad académica, a través de su apoyo para que encuentre en ella una fuente de inspiración y del empoderamiento para que el estudiante realice su propio aprendizaje en dicho contexto.

Con relación al apoyo social, Jairam y Kahl (2012) observaron que las tres fuentes de apoyo social (amistades académicas, familiares y docentes) actúan como soporte, mitigando el estrés del alumnado, y favoreciendo la finalización de los estudios. Por ello, los autores aconsejan a los doctorandos y doctorandas que construyan amistades en el ámbito académico, que busquen ayuda en miembros de la familia para la realización de ciertas tareas y que establezcan una relación profesional adecuada y activa con la persona que dirige su tesis doctoral.

Las emociones y los sentimientos experimentados en el proceso doctoral influyen en la satisfacción del alumnado respecto al programa de doctorado (Cheng, Taylor, Williams, \& Tong, 
2016), la cual está, a su vez, mediatizada por las expectativas. En relación con las emociones negativas, varios estudios demuestran que los problemas emocionales y motivacionales que experimenta el alumnado de doctorado a lo largo de los procesos de supervisión e investigación pueden determinar el fracaso y la deserción (Cotterall, 2013; Jairam \& Kahl, 2012).

En definitiva, a lo largo del proceso doctoral se pueden encontrar factores individuales y contextuales que favorecen o dificultan el desarrollo de competencias, afectando, asimismo a las emociones durante el proceso, las cuales, a su vez, influyen en el aprendizaje. De acuerdo con las investigaciones realizadas, aspectos personales tales como la motivación, la capacidad para organizarse y tomar decisiones y el bagaje previo actúan como favorecedores del proceso, en tanto que la ausencia de dichos elementos suponen barreras para el aprendizaje que pueden conducir al abandono (Castelló, Pardo, Sala-Bubaré, \& Suñe-Soler, 2017). Al mismo tiempo, elementos contextuales tales como una adecuada supervisión y el apoyo social, tanto en el ámbito académico como personal, se consideran facilitadores, mientras que una insuficiente e inadecuada supervisión y la ausencia de apoyo social dificultan la formación. La presencia de barreras se asocia con la aparición de emociones negativas, las cuales interfieren negativamente en el aprendizaje (Spaulding \& Rockinson-Szapkiw, 2012), aunque su efecto se puede mitigar gracias a los elementos facilitadores (Peltonen, Vekkaila, Rautio, Haverinen, \& Pyhältö, 2017).

A pesar de la importancia de estos elementos en la formación doctoral, hasta el momento, no se ha profundizado lo suficiente en el estudio de estos aspectos considerando en tiempo real la perspectiva de las personas doctorandas en los distintos momentos del proceso y centrándose en aquellos aspectos que son importantes en cada fase: expectativas en el inicio, dificultades, recursos y aprendizajes parciales en el proceso, y aprendizajes percibidos al final. Este es, por tanto, el objetivo de esta investigación.

\section{MÉTODO}

\section{Diseño}

Con el fin de cumplir los objetivos presentados, se ha llevado a cabo un estudio cualitativo, aplicando un cuestionario de preguntas abiertas a estudiantes en tres momentos de la formación doctoral, al inicio, durante el proceso y al final de los estudios.

Se comenzó recogiendo información en el curso 2013/2014 y se terminó la recogida en el curso 2017/2018. Se han considerado para el estudio aquellos casos que habían participado en la investigación en los tres momentos de su formación doctoral.

\section{Contexto}

El estudio se realiza en una universidad española centrándose en la formación de personas investigadoras delárea de Educación. En la investigación participa alumnado de dos programas 
de Doctorado, uno acorde con el Decreto 1393/2007 y otro acorde al Decreto 99/2011. El promedio de estudiantes de nuevo ingreso en ambos programas durante los años de recogida de información de la investigación es de 10.5.

La caracterización del alumnado de ambos programas de Doctorado es similar. El nivel socioeconómico es medio-alto, habiendo un predominio de mujeres (67\%). En cuanto a la edad, el rango está comprendido entre 25 y 56. Un alto porcentaje (33\%) de estudiantes es internacional, habiendo gran diversidad respecto al lugar de procedencia llegando a contar 15 nacionalidades diferentes, y con un alto predominio de países de América Latina. La gran mayoría del alumnado extranjero cuenta con algún tipo de ayuda para financiar sus estudios.

\section{Participantes}

Con el fin de cumplir el objetivo, se invitó al alumnado a aportar información en tres momentos del proceso doctoral, al inicio, durante el proceso y al final, es decir, una vez defendida la tesis doctoral. No obstante, no todo el alumnado completó los tres cuestionarios, debido entre otras, a las siguientes razones: (a) comienzo del doctorado con anterioridad al curso en el que se inició esta investigación; (b) abandono de los estudios de doctorado; (c) interrupción de la participación en la investigación.

Fueron ocho las personas que cumplieron los criterios de inclusión como muestra participante en el estudio, cinco mujeres y tres hombres. Su rango de edad al iniciar los estudios estaba entre 25 y 47 años. Los estudios universitarios que les dieron acceso al doctorado fueron filología, comunicación, educación, pedagogía, física e historia. Cuatro personas pertenecían a un programa de doctorado regulado por el Decreto 1393 de 2007, mientras que los cuatro restantes cursaron el programa de doctorado regulado por el Decreto 99 de 2011.

\section{Instrumento}

La información ha sido recogida a través de tres cuestionarios de preguntas abiertas respondidos al inicio, en el proceso y al final. Estos cuestionarios están basados en los guiones de entrevistas aplicados y validados en una investigación anterior llevada a cabo con el mismo colectivo de estudiantes (Villardón-Gallego \& Yániz, 2013). Una vez diseñados, los cuestionarios fueron revisados y avalados por una comisión de evaluación conformada por tres doctores especialistas en investigación en educación, dos de ellos miembros del claustro del programa de doctorado.

Los tres cuestionarios completos incluían una parte destinada a recoger información sobre características sociodemográficas y académicas, tales como edad, sexo y formación de acceso. Además, contenían preguntas dirigidas a conocer distintos aspectos de su recorrido doctoral. De acuerdo con lo pretendido en este estudio, se han seleccionado para su análisis las siguientes preguntas: 
- En el Cuestionario inicial (CI) se analizaron las siguientes siete cuestiones centradas en las expectativas: (1) ¿Qué esperas del doctorado? (2) ¿Qué crees que vas a aprender en el doctorado? (3) ¿Cómo crees que va a ser el proceso de realización de tesis doctoral? (4) ¿Qué dificultades esperas encontrar y cómo esperas resolverlas? (5) ¿Qué factores, elementos, aspectos crees que te van a ayudar a vencer las dificultades durante la investigación doctoral? (6) Señala tus puntos fuertes para llevar a cabo una investigación; (7) Señala tus puntos débiles para llevar a cabo una investigación.

- En el Cuestionario de proceso (CP) se consideraron cuatro preguntas sobre dificultades, apoyos y aprendizajes intermedios: (1) ¿Qué crees que estás aprendiendo en el doctorado? (2) ¿Cómo crees que está siendo el proceso de realización de tesis doctoral? (3) ¿Qué dificultades has encontrado y cómo las has resuelto? 4) ¿Qué factores, elementos, aspectos te han ayudado a vencer las dificultades durante la investigación doctoral?

- En el Cuestionario final (CF) se analizaron dos preguntas relacionadas con los aprendizajes percibidos: (1) ¿Qué aprendiste en el doctorado? (2) ¿A partir de tu experiencia, qué formación crees necesaria para realizar la tesis doctoral?

\section{Procedimiento}

Al inicio de los estudios de doctorado se explicaba al alumnado el objetivo de la investigación y el carácter confidencial de la información recogida, solicitando su participación voluntaria. Con el fin de proteger el anonimato, el nombre de las ocho personas participantes se ha modificado por un seudónimo. Se recogió información anualmente desde el 2013 hasta el 2017, comenzando con el CI, siguiendo con el CP y terminando con el CF. Se da la circunstancia de que, dependiendo de la duración del recorrido doctoral, algún participante completó más de un CP. En tal caso, se ha seleccionado para el análisis aquel cuestionario contestado en la mitad del proceso, considerando el tiempo transcurrido desde el inicio hasta el final.

\section{Análisis de datos}

Las respuestas a los cuestionarios han sido analizadas con la ayuda del programa Maxqda, identificando las expectativas, dificultades, apoyos, emociones y aprendizajes percibidos de cada uno de los ocho participantes.

Para realizar el análisis de las respuestas a cada una de las preguntas anteriores se han establecido dos tipos de categorización diferentes, una referida a los Factores y la otra a los Aprendizajes Percibidos. La categorización de Factores se ha realizado por medio de un análisis inductivo, esto es, basándose en el contenido de las respuestas emitidas por las personas participantes, dando como resultados subcategorías como Dificultades, Recursos y Apoyos, Emociones positivas y Emociones negativas. 
En la categoría de Aprendizajes Percibidos se ha realizado un análisis deductivo, tomando como referencia las competencias del perfil de egreso marcadas por el Real Decreto 99/2011.

Dos investigadoras realizaron individualmente la categorización de las respuestas. Después del proceso de categorización individual, ambas investigadoras analizaron y discutieron coincidencias y discrepancias hasta llegar a consenso.

\section{RESULTADOS}

Las ocho personas participantes han indicado al inicio sus expectativas en torno al aprendizaje, dificultades y recursos; durante el proceso, los sentimientos y emociones que han experimentado, las dificultades y apoyos, así como los aprendizajes parciales. Al final de la formación doctoral, se han recogido los aprendizajes percibidos. La Tabla 1 incluye un resumen de los resultados.

A continuación, se describe de forma detallada la información aportada por cada una de las personas participantes en los tres momentos del proceso de formación.

\section{María}

En cuanto a las expectativas de aprendizaje, en el inicio de los estudios, María espera desarrollar la competencia CB1, es decir, aspira a llegar a una comprensión sistémica de su campo de estudio y dominar los métodos de investigación, así como a diseñar y llevar a cabo una investigación (CB2).

Ya en el comienzo de su formación, María incluye la idea del impacto social de su tesis doctoral, aprendizaje que se vincula con la competencia propia del programa de doctorado cursado CB7:

“... podré aportar ideas de cara a posibles mejoras en los planes de estudio de la formación inicial del profesor"(María, i24)

Indica, asimismo, que espera realizar este aprendizaje en equipo, haciendo referencia a una de las capacidades personales marcadas por el Decreto, relacionada con el trabajo en equipo y de forma autónoma (CA4).

A nivel emocional siente miedo a la vez que está motivada por comenzar los estudios. De hecho, considera que el "entusiasmo y las ganas de aprender" (María, i44) son sus puntos fuertes.

Es consciente de que la formación doctoral conllevará tanto momentos buenos como difíciles. Las principales dificultades esperadas están relacionadas con la disponibilidad y la gestión del tiempo. Entre los elementos que van a facilitar el éxito cita la constancia, la fuerza de voluntad y el apoyo social, fundamentalmente en la directora de la tesis.

En el proceso, María es consciente de la adquisición de aprendizajes relacionados con la competencia CB1, tales como buscar información y analizar datos, y con la CB4, como interpretar resultados, así como con trabajar en equipo (CA4). 


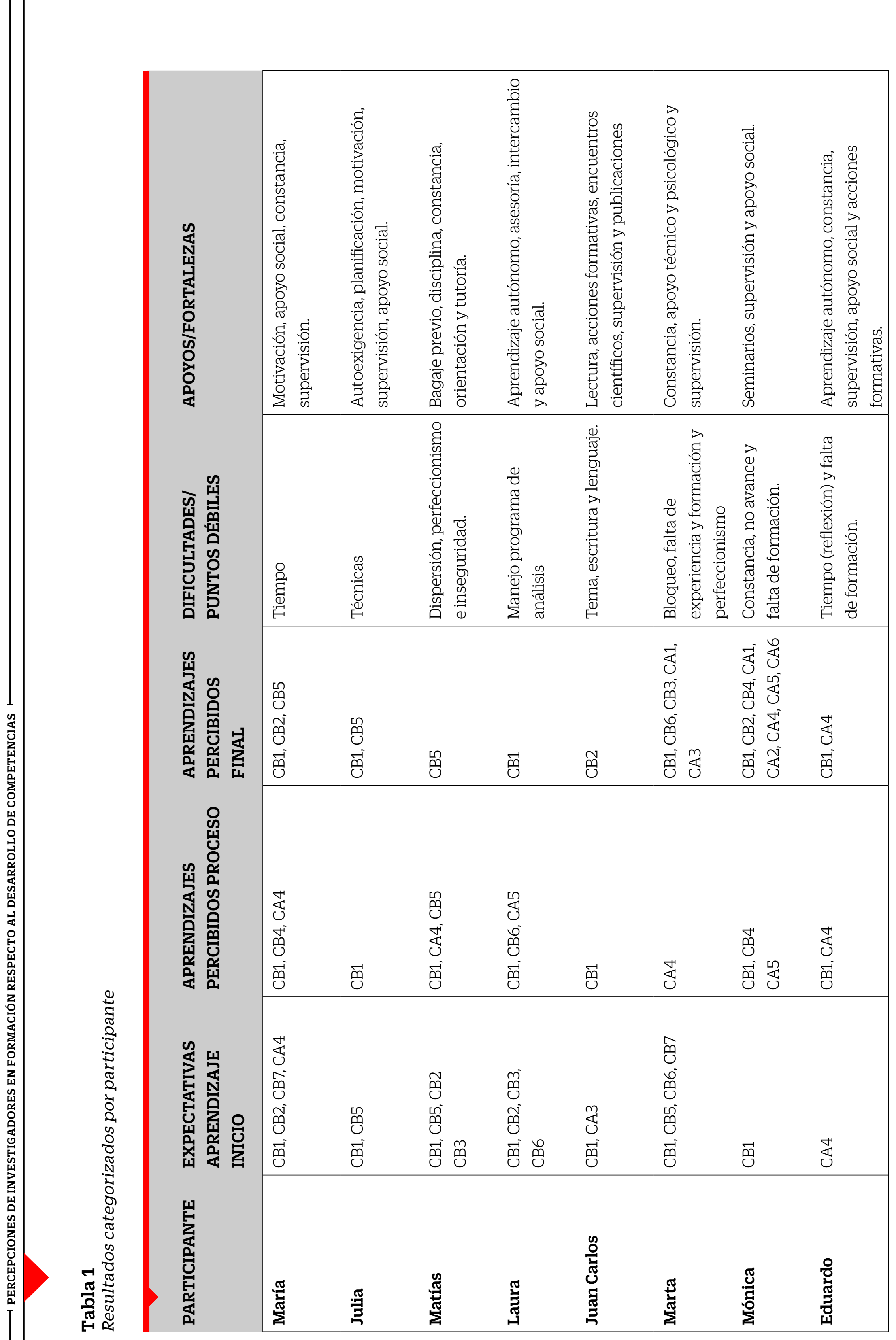


Las dificultades se van resolviendo con la supervisión de su directora, el apoyo social y la motivación que tiene por la investigación.

“La motivación que tengo, el apoyo inmenso y el sentirme acompañada continuamente en el proceso." (María, p46)

Una vez finalizados los estudios de doctorado, María considera que ha desarrollado aprendizajes relacionados con la competencia CB1, tales como "buscar información relevante sobre un tema en bases de datos", "análisis cualitativos", con la competencia CB2, referida a la capacidad para diseñar una investigación, y con la competencia CB5, relacionada con la capacidad de comunicación con la comunidad científica, a través de la "publicación de artículos".

\section{Julia}

Julia inicia el programa de doctorado con unas expectativas altas en relación al aprendizaje, vinculadas al desarrollo de la competencia CB1 y CB5.

Anticipa dificultades relacionadas con aspectos técnicos de la metodología de investigación, un aspecto que valora como débil en su bagaje. Espera superarlas solicitando ayuda y, de forma autónoma, a través de la lectura. Entre sus puntos fuertes para lograr el éxito cita la motivación por aprender, la disciplina y el orden, la exigencia y la capacidad de planificación. Ella percibe que esta formación irá entrelazándose con su vida personal y diversos aspectos emocionales. Además, la doctoranda remarca la necesidad del apoyo de su directora de tesis y de otras personas académicas cuando lo crea necesario.

Durante el proceso, Julia es consciente del gran aprendizaje que está realizando, fundamentalmente en la competencia CB1:

¡i TODO!! cómo investigar, el porqué de una investigación" (Julia, p37-39)

Este proceso de aprendizaje cursa con altibajos emocionales. Ha confirmado sus limitaciones técnicas debidas a su escaso bagaje investigador, que intenta resolver a través de la formación y las interacciones con los miembros de su equipo y su directora.

"Muchas dificultades, al no poseer un bagaje investigador te ves limitado a entender y resolver cosas (...) Mis compañeros de equipo y mi directora de tesis me ayudan a resolverlas, también la asistencia a formaciones." (Julia, p42-43)

Finalizada la formación doctoral, Julia señala que los conocimientos adquiridos están relacionados con la competencia CB1, además de con la docencia, que podrían vincularse con la competencia CB5.

\section{Matías}

Las expectativas de aprendizaje de Matías al inicio de la formación doctoral incluyen conocimientos relacionados con la competencia CB1 y CB5. Hace mención a la elaboración de un proyecto riguroso (CB2) y a la originalidad (CB3).

“... ordenar mis ideas en un proyecto de investigación que sea sólido científicamente, riguroso académicamente y un poco original." (Matías, i38-40) 
Entre las dificultades que espera para cubrir las expectativas, menciona la falta de formación académica y en metodología de investigación. Además, hace referencia a la necesidad de convergencia entre sus intereses académicos y la tesis. Por otro lado, menciona su preocupación por la financiación de la investigación doctoral.

“... que no haya mucha divergencia entre mis intereses académicos y la investigación de

la tesis. Dificultades de tipo económico: tener la suficiente financiación (Matías, i48-49)

A nivel personal, afirma que puede llegar a ser excesivamente perfeccionista, lo que le lleva a sentirse inseguro e insatisfecho con el resultado. Asimismo, anticipa dificultades para seguir el proyecto acordado, ya que se le ocurren muchas ideas que le llevan a dispersarse.

A pesar de ello, reconoce elementos facilitadores que le podrán ayudar a finalizar el trabajo doctoral, como es la tutorización y orientación de personas académicas, la capacidad de lectura y de redacción y su experiencia previa en investigación, así como la disciplina y la constancia.

Durante el proceso considera que está aprendiendo metodología, aprendizajes relacionados con la competencia CB1, además de aprender a trabajar en equipo (CA4) y a escribir y difundir sus hallazgos en publicaciones y reuniones científicas (CB5).

“A conocer mejor todos los distintos aspectos de una investigación. A trabajar en equipo y compartir dudas (metodológicas, prácticas...). A descubrir el mundo de los congresos, las publicaciones (...) A escribir ... para comunicarlo a los demás" (Matías, p35-39)

La principal dificultad que Matías no ha logrado superar es la falta de tiempo de dedicación a la tesis doctoral, tanto por razones personales como profesionales.

Al finalizar su tesis doctoral, Matías reconoce haber aprendido sobre todo escritura académica (CB5).

“... sobre todo escritura académica, a elaborar un texto en un género concreto como es una tesis doctoral." (Matías, f34)

\section{Laura}

Al iniciar su recorrido doctoral, Laura espera aprender conocimientos vinculados a CB1 y CB2. “Diseñar, planificar, decidir, analizar, etc." (Laura, i34)

No se plantea tanto confirmar unas hipótesis sino sorprenderse con los resultados, expectativa que se puede relacionar con las competencias CB3 y CB6.

Las expectativas emocionales son mixtas, piensa que este proceso le llevará a vivir emociones positivas, pero que estará lleno de contrariedades.

“Un proceso de alegrías y dificultades... la alegría de avanzar en una investigación... Por otra parte, las dificultades o contrariedades que... vayan surgiendo." (Laura, i36)

Las dificultades señaladas tienen que ver con el propio proceso investigador, como por ejemplo, no tener en cuenta factores intervinientes que pueden afectar a los resultados o no lograr una visión global de la investigación. 
Entre los elementos facilitadores del contexto, menciona la supervisión, la orientación y el trabajo en equipo, así como lectura de investigaciones sobre el tema. A nivel personal, identifica como puntos fuertes para llevar a cabo la investigación, la meticulosidad, la capacidad de planificación, la motivación e interés.

“La lectura previa de investigaciones anteriores realizadas en ese mismo ámbito de estudio y el poder compartir el conocimiento con personas que se encuentren trabajando en esas mismas líneas." (Laura, i38)

Durante el proceso, Laura señala que está adquiriendo capacidades básicas relacionadas con el área de conocimiento y con el proceso investigador vinculadas a la competencia CB1. Asimismo, valora la importancia de la transferencia del conocimiento, locual se puederelacionar con CB6, y de la multidisciplinariedad, aspecto que se relaciona con CA5.

“...enriqueciendo mi visión con el aporte de diferentes paradigmas y autores; por

otro lado, estoy conociendo diferentes técnicas y metodologías; y ... la importancia de la transferencia de conocimiento y de la multidisciplinariedad..." (Laura, p34)

La dificultad más importante que se ha encontrado está relacionada con el manejo de un programa de análisis de información cuantitativa, debido a la falta deformación previa al respecto. Supera dicha dificultad pidiendo asesoramiento y de forma autodidacta, leyendo manuales.

Los elementos facilitadores que menciona en el proceso son el apoyo social de las personas que están en una situación similar, las acciones formativas y los seminarios donde intercambiar ideas y sugerencias.

Una vez terminado el proceso, Laura señala haber desarrollado habilidades y conocimientos propios de la investigación (CB1).

"Por una parte, desarrollar habilidades necesarias en la formación investigadora; por otra parte, ampliar mis conocimientos sobre el tema tratado." (Laura, f32)

\section{Juan Carlos}

En el comienzo del doctorado, Juan Carlos espera realizar aprendizajes vinculados a CB1, tales como la adquisición de herramientas para llevar a cabo la propuesta de investigación. Igualmente, espera aprender gestión del conocimiento, de la innovación y de la investigación (CA3).

En estos momentos iniciales, su mayor preocupación es la aceptación del tema que propone para investigar. Para superar esta dificultad es consciente de la necesidad de una buena fundamentación y justificación teórica.

“... fundamentaré el proyecto con los antecedentes históricos de los problemas.” (Juan Carlos, i40)

Los factores facilitadores con los que cuenta son el profesorado, el tutor y la dedicación a tiempo completo.

A lo largo del proceso, considera que está aprendiendo las habilidades y conocimientos relacionados con la investigación (CB1). 
“... procesos de investigación en diferentes contextos, así como a analizar instrumentos desde una perspectiva cuantitativa." (Juan Carlos, p36)

La dificultad más importante en estos momentos esta relacionada con la escritura, dificultad que intenta superar a través de lecturas científicas. Otros elementos facilitadores del proceso de aprendizaje son:

"El asesoramiento de los directores de tesis; la lectura científica; los cursos académicos; el intercambio en los seminarios y participación en congresos y publicación en revista científica." (Juan Carlos, p40-42)

En estos momentos el doctorando tiene la sensación de que la tesis ocupa el centro de su vida, lo que le permite avanzar de forma exitosa en este largo camino.

“...se está convirtiendo en mi vida... la dedicación completa... me está facilitando su realización..." (Juan Carlos, p40-42)

Al terminar la formación, Juan Carlos afirma haber aprendido a llevar a la práctica una investigación (CB2) y a ser investigador, lo que se vincula con la adquisición de la identidad como investigador, proceso que implica el desarrollo de diversas competencias del perfil de egreso.

\section{Marta}

Las expectativas de aprendizaje de Marta al iniciar el doctorado no solo se relacionan con habilidades propias de la investigación (CB1), sino que también espera aprender habilidades para manejarse en el mundo académico. Aunque esta afirmación puede relacionarse con varias competencias del perfil de egreso, se vincula más específicamente con la CB5 y CB6.

“Cómo investigar, qué es investigar, cómo explotar las fuentes de información y los datos, cómo publicar y dónde, aprender dónde buscar, metodología... (Marta i34)

A nivel afectivo, las expectativas de Marta son bastante negativas. Considera que el proceso será duro, solitario y largo, en el que será necesario la constancia en aras de una mayor calidad de la investigación (CB7).

"Duro y solitario. Mucho tiempo por delante y hay que ser constante. De la persistencia del doctorando depende la calidad de los resultados de la investigación." (Marta, i36)

Entre las dificultades esperadas, Marta considera que el doctorado supone mucho trabajo y espera tener momentos de bloqueo, debido a la falta de experiencia.

Para superar estas dificultades la doctoranda contará con la supervisión y orientación de su directora y otras personas expertas. A nivel personal, valora la motivación como elemento fundamental para seguir avanzando.

"En esos momentos de bloqueo (...) hay veces que es necesario que un experto te oriente (...) En este punto, el grado de motivación es fundamental." (Marta, i40)

Durante el proceso, Marta señala aprendizajes relacionados con la competencia para aprender que le permitirá convertirse en una buena investigadora (CA4), percepción que enlaza con el desarrollo de la identidad investigadora. 
Las dificultades encontradas tienen que ver sobre todo con la falta de formación investigadora (herramientas y programas de análisis de información). Afronta estas dificultades de forma autónoma, buscando ayuda en Internet, y con el apoyo de miembros del equipo y de la dirección.

En la parte emocional, considera que el proceso es enriquecedor, pero también solitario. Durante la formación, piensa que se está haciendo más perfeccionista, lo que le lleva a no estar satisfecha con lo que logra. No obstante, valora positivamente el apoyo técnico y social con el que cuenta.

“El apoyo psicológico de aquellos que han estado a mi lado, tanto en el trabajo como en casa, han sido piezas clave para superar las dificultades emocionales que han ido surgiendo durante la investigación." (Marta, p40)

Al finalizar el doctorado, Marta considera que ha aprendido mucho, tanto a nivel de conocimiento del área y habilidades para la investigación (CB1; CB6), como aspectos más transversales (CB3; CA1; CA3):

“.. metodología de investigación, recursos digitales, cómo funcionan las revistas científicas, técnicas de aplicación de mi investigación, (...) y (...) a organizarme, tener recursos ante problemas que surgen durante la investigación, gestión del tiempo, gestión de recursos, génesis de ideas, (...). Y muchas otras relacionadas con la materia de mi trabajo." (Marta, f34)

\section{Mónica}

Mónica inicia el doctorado con la expectativa de adquirir conocimientos relacionados con el proceso investigador (CB1). La doctoranda considera que es un proceso complejo y con importantes dificultades a vencer, a la vez que gratificante, en el que se avanza poco a poco.

“Creo que va a ser un proceso duro, con dificultades y obstáculos, pero también

gratificante y ... enriquecedor. (...)... va a ser así, con altibajos, con muchas dudas y con pequeños pasos que poco a poco cogerán forma en una unidad con sentido." (Mónica, i39)

Entre sus puntos de mejora, indica la necesidad de trabajar de forma autónoma y de planificarse bien:

“..es muy importante organizarse bien, establecer objetivos y plazos a corto plazo, planificar el progreso de la tesis." (Mónica, i43-44)

La doctoranda identifica varios aspectos facilitadores del proceso, algunos organizativos y otros de tipo social:

“... el apoyo de mi tutora y el equipo de investigación son muy importantes... El apoyo de mi familia y mis amigos también es indispensable... es muy importante tener personas a las que poder contarles cómo vas y las dificultades..." (Mónica, i43-44)

Durante el proceso, Marta percibe aprendizajes vinculados con la investigación y el área de conocimiento, tales como metodología, sociología y estadística (CB1). Sin embargo, es consciente de otros aprendizajes que ha desarrollado durante el proceso tales como tomar 
decisiones con criterio, capacidad de síntesis, inferencia de resultados (CB4), a la vez que se ha hecho consciente del carácter abierto y dinámico de la investigación (CA5) .

A Mónica, el proceso le está resultando duro y gratificante a la vez, está perdida en algunos momentos, en tanto que en otros camina en la dirección deseada. Todo ello le lleva a sentir tanto frustración y soledad, como satisfacción cuando hay avances.

"Duro cuando te sientes perdida... porque a veces te sientes sola y frustrada porque las cosas no salen como esperabas. Sin embargo, se aprende a hacer frente a las adversidades y buscar soluciones justificadas y con criterio. A través del trabajo, de consultar distintas fuentes, (...) se va avanzando. Es gratificante cuando vas caminando en la dirección deseada." (Mónica, p36)

Las dificultades tienen que ver con el desconocimiento de algunos temas y de metodología (programas de análisis deinformación), para cuyo afrontamientola lectura ha sidofundamental, así como la ayuda de la tutora y de los y las compañeras. Además, menciona otros factores facilitadores del proceso como los seminarios del equipo de investigación, donde presentar y compartir experiencias, y cualidades personales como la constancia, la serenidad y la fortaleza.

Al finalizar su formación doctoral, Mónica es consciente de haber logrado diversos aprendizajes vinculados con las competencias CB1 y CB2. Afirma haber desarrollado identidad investigadora, lo que, en su opinión, incluye, además de la adquisición de destrezas específicas vinculadas con la investigación, la consciencia de la complejidad de la realidad, de la necesidad de ser flexible, la capacidad para reflexionar, para tomar decisiones con criterio y la necesidad de seguir aprendiendo, adquisiciones que se pueden vincular con las competencias CB4, CA1, CA2, CA4, CA5 y CA6.

“... he comprendido la complejidad de la realidad y la necesidad de ser flexible y abierta, he adquirido mayor capacidad de reflexión, he conocido distintas metodologías y técnicas de investigación, (...) En este camino he ido adquiriendo conocimientos y habilidades para tomar decisiones con criterio y hacer frente a las dificultades... He comprendido que este es un proceso de aprendizaje continuo y que es solo el principio sobre el que tengo que seguir trabajando y aprendiendo en un futuro como investigadora." (Mónica, f34)

\section{Eduardo}

En el inicio del doctorado, Eduardo espera que el proceso sea estimulante y le permita adquirir herramientas para el desarrollo profesional y académico (CA4).

El doctorando está muy motivado, por lo que espera un recorrido gratificante, aunque no exento de dificultades, vinculadas sobre todo con aspectos técnicos de la investigación. No obstante, reconoce que posee elementos que le ayudarán a superar las barreras:

“El trabajo personal, las instrucciones de los cursos y seminarios del doctorado, la ayuda del tutor..." (Eduardo, i39) 
Durante el proceso, Eduardo se reafirma en que la formación doctoral es muy enriquecedora, ya que está aprendiendo muchas cosas, entre ellas, habilidades propias de la investigación (CB1) y otras destrezas relacionadas con la autogestión del aprendizaje (CA4):

“... estoy aprendiendo a mejorar la gestión y aprovechamiento del tiempo, estoy mejorando mi organización y orden a la hora de trabajar. Por otro lado, desde el punto de vista de los contenidos, estoy formándome en aspectos relativos al ámbito de la investigación..." (Eduardo, i39)

Entre las dificultades, menciona que en ocasiones le falta tiempo para reflexionar, ya que el proceso va demasiado rápido. Otras dificultades que está experimentando tienen que ver con su escasa formación en metodologías de investigación. Para superarlas cuenta con los cursos de doctorado y otras actividades formativas, así como con la lectura y el asesoramiento de la directora y otras personas expertas, y con el apoyo social de los y las compañeras.

"El hecho de compartir despacho con compañeros inmersos en el mismo proceso me ha ayudado mucho, ya que puedes aprender de ellos... tener su apoyo, (...) en metodología y análisis de resultados es donde más dificultades he tenido. En este punto he recurrido a expertos, así como a la lectura, a la tutora y a los compañeros." (Eduardo, p39)

Cuando finaliza la tesis, Eduardo reconoce haber aprendido tanto del área de conocimiento como de metodología de investigación (CB1). Resalta otros aprendizajes que tienen que ver con un cambio de identidad, que implica un cambio actitudinal y comportamental (CA4):

“...te obliga a ser perseverante, aplicado, metódico (...). A nivel conceptual creo que la inmersión en el campo, sobre todo, de la metodología ha sido muy profunda... la inmersión, también, en el área de conocimiento ha sido muy importante. Pero me gustaría subrayar, además, lo actitudinal (Eduardo, f31-32)

\section{DISCUSIÓN}

En esta investigación se ha analizado el proceso de formación doctoral a partir de la información recogida en distintos momentos del proceso. Se organiza la discusión en torno a los dos ejes principales: aprendizaje y factores, tanto facilitadores como obstaculizadores, del proceso.

\section{Aprendizaje}

Las expectativas de la totalidad de participantes se centran en el desarrollo de competencias para la investigación, relacionadas con el manejo de técnicas y herramientas y con la profundización en su área de conocimiento (CB1), aprendizaje que consideran haber logrado al finalizar el doctorado, lo que se puede estimar como un éxito de los programas. En verdad, durante el doctorado se aprende a investigar, diseñando y desarrollando una investigación, en torno a la cual, se realizan otros aprendizajes. Se confirma la validez del aprendizaje basado en 
investigación en la formación doctoral (Villardón-Gallego, 2015).

El resto de aprendizajes vinculados a otras competencias incluidas en el perfil de egreso del doctorado no es tan unánimemente percibido por los y las participantes. Así, tres estudiantes mencionan haber avanzado en la capacidad para comunicarse en la comunidad científica, a través de la escritura de artículos y presentación en foros y reuniones científicas (CB5), de los cuales dos, Matías y Julia, lo señalaron al inicio como expectativa de aprendizaje.

Son tres también los estudiantes que consideran haber aprendido a diseñar y desarrollar un proyecto de investigación (CB2). Tan solo una estudiante, Marta, hace mención a la adquisición de la capacidad para fomentar avances en la sociedad del conocimiento (CB6), aprendizaje que había planteado en sus expectativas iniciales. En la misma línea, esta estudiante declara haber desarrollado capacidades para contribuir a avanzar en el conocimiento a partir de una investigación original (CB3). Es preocupante que solo una participante perciba este logro, ya que la formación doctoral debe capacitar para mejorar la sociedad produciendo y transfiriendo nuevo conocimiento (Baptista, Frick, Holley, Remmik, \& Tesch, 2015)

Finalmente, Mónica es la única estudiante que reconoce haber realizado aprendizajes relacionados con el análisis crítico, con la evaluación y la síntesis de ideas nuevas y complejas (CB4), aprendizaje que ya percibió durante el proceso formativo.

Es todavía menos frecuente al finalizar el doctorado la percepción de aprendizajes relacionados con la adquisición de capacidades y destrezas personales. Dos estudiantes, Eduardo y Mónica, reconocen haber aprendido a trabajar tanto en equipo como de manera autónoma (CA4), un aprendizaje que estaba entre las expectativas de aprendizaje de Eduardo y respecto al que ya percibió avances en el proceso. Mónica, además, reconoce adquisiciones relacionadas con otras destrezas incluidas en CA1, CA2, CA5 y CA6, algunos de los cuales ya había mencionado en el proceso.

Estos resultados indican que el alumnado participante cuando termina el doctorado no es consciente de haber desarrollado muchos de los aprendizajes relacionados con las competencias básicas marcadas como finalidades educativas por el Decreto y que corresponden a competencias transversales, disposiciones, conductas y metacompetencias, en términos de Durette et al. (2014). No se puede decir que no hayan adquirido en mayor o menor medida dichos aprendizajes, pero parecen no tener demasiada conciencia de ello, lo cual podría estar relacionado con las concepciones de la investigación que tienen (Álvarez-Álvarez, Elexpuru, Castelló, Villardón-Gallego, \& Yániz, 2017). Dado que la conciencia de las propias capacidades es importante para el desempeño de la mismas (Lunenburg, 2011), se considera fundamental que los programas de doctorado trabajen en esta dirección.

Según la información obtenida, parece que las expectativas de aprendizaje iniciales influyen en la percepción de aprendizajes al final; es decir, aquellos estudiantes que esperan aprender ciertas habilidades son más conscientes de haberlas logrado. Estos resultados 
coinciden con los obtenidos por Combs y Onwuegbuzie (2012) con respecto a un curso de doctorado sobre estadística. Asimismo, los aprendizajes percibidos en el proceso se relacionan con los aprendizajes percibidos al final.

Por ello, se recomienda que los programas diseñen actividades específicas de reflexión compartida para hacer conscientes a los y las estudiantes de que la formación doctoral capacita para un desempeño altamente cualificado, no solo en cuanto a técnicas de investigación sino también con respecto a la contribución del conocimiento, a la mejora de la sociedad, a la capacidad para la innovación, etc. Estas actividades, en sí mismas, serían una herramienta para el desarrollo de las competencias transversales, de comportamientos y de actitudes propias de los y las investigadoras. De esta manera, se lograría mejorar el desempeño de las personas investigadoras recién doctoradas desarrollando durante el proceso formativo su identidad investigadora desde un concepto amplio, como contribución a la sociedad y al conocimiento y como desarrollo personal.

Hay que tener en cuenta que la recogida de información fue a través de preguntas abiertas, lo que puede haber incidido en que no aparezca un mayor número de aprendizajes percibidos. No obstante, se considera que esta modalidad de pregunta permite recoger de forma más certera su consciencia sobre los aprendizajes realizados, ya que no se incluye ninguna sugerencia de posible respuesta.

Por otro lado, en la interpretación de los resultados hay que ser conscientes de que en las competencias y capacidades incluidas en el Decreto que regula la formación doctoral hay cierto solapamiento inevitable, lo que dificulta la categorización excluyente de las respuestas del alumnado con respecto a los aprendizajes percibidos.

\section{Factores}

Los resultados confirman la importancia durante el proceso doctoral de la dirección, tanto por la supervisión técnica como por el apoyo social, ya que la totalidad de los y las participantes lo mencionan como recurso para vencer las dificultades. En consecuencia, tanto la sincronía entre tutor o tutora y estudiante, como una supervisión basada en las necesidades del alumnado (De Kleijn, Meijer, Brekelmans, \& Pilot, 2015),que establezca una comunicación abierta y negociadora, y que preste ayuda continua al desarrollo de competencias y de oportunidades de carrera, son elementos fundamentales en la formación doctoral (Duke \& Denicolo, 2017).

Por tanto, es necesario que las personas directoras estén altamente cualificadas para realizar una supervisión y acompañamiento de calidad. Con este fin, se sugiere su participación en actividades de formación y encuentros que potencien su compromiso con su desarrollo profesional, mejorando continuamente sus competencias para la supervisión (Duke \& Denicolo, 2017). 
Asimismo, coincidiendo con otras investigaciones (Gardner, Jansujwicz, Hutchins, Cline, \& Levesque, 2012; Spaulding \& Rockinson-Szapkiw, 2012) se confirma la importancia del apoyo social tanto por parte de los compañeros y compañeras de doctorado como de la familia y amistades. De hecho, el apoyo social y la búsqueda de ayuda se encuentra entre las estrategias de afrontamiento que facilitan el aprendizaje (Combs \& Onwuegbuzie, 2012) y disminuyen el burnout y el abandono (Peltonen et al., 2017). Es por ello que se sugiere incluir en los programas de doctorado acciones formativas y espacios que favorezcan el intercambio de experiencias, para fomentar la creación de comunidades de aprendizaje entre personas investigadoras.

El alumnado valora positivamente las actividades formativas realizadas dentro de los programas, tanto los cursos específicos sobre diferentes técnicas y metodologías de investigación como la participación en encuentros científicos y en publicaciones, que ofrece, al igual que la realización de la tesis doctoral, la oportunidad de aprender activamente de la experiencia bajo la guía y supervisión del director o directora. Por otro lado, las lecturas son un recurso fundamental en un proceso de aprendizaje autónomo.

El modelo de Leech (2012) considera importantes en la formación del personal investigador en educación ciertas características personales. En esta misma línea, siete participantes citan la constancia y "autoexigencia", seis de ellos la mencionan como una cualidad, y una participante, Mónica, como un aspecto por mejorar en ella. Asimismo, dos estudiantes citan la motivación como una variable importante para el éxito en los estudios.

Por el contrario, el perfeccionismo es reconocido por dos participantes como una barrera, ya que les lleva a sentirse inseguros de lo que están haciendo y, por tanto, a bloquearse. Para otro estudiante, la dispersión es una dificultad que le impide enfocarse en la investigación.

La dificultad más comúnmente percibida en este grupo de investigadores es la falta de formación y de bagaje previo en métodos y técnicas de investigación y en escritura y lenguaje académico. Por tanto, las actividades formativas centradas en metodologías, técnicas y herramientas de investigación son fundamentales para desarrollar competencias instrumentales necesarias tanto para realizar la tesis doctoral como para la realización de futuras investigaciones.

Con respecto a la dimensión emocional, los y las participantes son conscientes de la presencia durante el doctorado de emociones positivas y negativas, dependiendo de los avances o de los bloqueos, confirmando lo encontrado en otras investigaciones (Coterall, 2013; McLaughlin, 2003). Es importante para los y las investigadoras en fomación hacerse conscientes de las emociones y de cómo estas afectan al aprendizaje, como un primer paso para su autorregulación (Villardón-Gallego \& Yániz, 2014).

En definitiva, durante el proceso doctoral el alumnado comienza a desarrollar su identidad investigadora (McAlpine \& Lucas, 2011), lo cual supone tanto la adquisición de competencias relacionadas con la investigación, el trabajo autónomo y en equipo, como el 
desarrollo de unos valores y actitudes relacionados con el rigor, el compromiso social y la ética de la investigación. Además, como parte de esta identidad, las personas investigadores deben adquirir conciencia de pertenencia a la comunidad científica (Kobayashi, Mcginn, Pechar, y Wisker, 2015; McAlpine y Amundsen, 2009), lo cual implica contribuir de forma activa al avance del conocimiento y a su transferencia para la mejora de la sociedad (Reale et al., 2017). Para ello, es fundamental que se integren en un equipo de investigación y que participen en publicaciones y encuentros científicos y de difusión.

Esta investigación ha permitido conocer los aspectos que rodean el desarrollo de competencias propias de la formación doctoral a través de las reflexiones a lo largo del proceso de ocho personas investigadoras que culminaron con éxito sus estudios para, en base a los resultados, proponer sugerencias de mejora. No obstante, sería interesante investigar y analizar el proceso en aquellos estudiantes que no llegaron a terminar su formación, para conocer las causas del fracaso y diseñar medidas para paliarlas.

\section{REFERENCIAS}

Álvarez-Álvarez, M., Elexpuru, I., Castelló, M., Villardón-Gallego, L., \& Yániz, C. (2017). The "why" and "what for" of research in Social Sciences: Early career researchers'conceptions. Electronic Journal of Research in Educational Psychology, 15(3), 598-623. doi: http://dx.doi.org/10.14204/ejrep.43.16098

Anderson, T. (2017). The doctoral gaze: Foreign PhD students' internal and external academic discourse socialization. Linguistics and Education, 37, 1-10. doi: https://doi.org/10.1016/j.linged.2016.12.001

Baptista, A., Frick, L., Holley, K., Remmik, M., \& Tesch, J. (2015). The doctorate as an original contribution to knowledge: Considering relationships between originality, creativity, and innovation. Frontline Learning Research, 3(3), 51-63. doi: https://doi.org/10.14786/flr.v3i3.147

Cantwell, R. H., Scevak, J. J., Bourke, S., \& Holbrook, A. (2012). Individual Differences That Affect the Quality of Learning in Doctoral Candidates. En J. R. Kirby \& M. J. Lawson (Eds.), Enhancing the Quality of Learning (pp. 93-114). Cambridge: Cambridge University Press. doi: https://doi.org/10.1017/CBO9781139048224.007

Castelló, M., Pardo, M., Sala-Bubaré, A., \& Suñe-Soler, N. (2017). Why do students consider dropping out of doctoral degrees? Institutional and personal factors. Higher Education, 74(6), 1053-1068. doi: https://doi.org/10.1007/ s10734-016-0106-9

Castelló, M., Kobayashi, S., Mcginn, M. K., Pechar, H., Vekkaila, J., \& Wisker, G. (2015). Researcher Identity in Transition : Signals to Identify and Manage Spheres of Activity in a Risk-Career. Frontline Learning Research, 3(3), 35-50. doi: https://doi.org/10.14786/flr.v3i3.149 
Cheng, M., Taylor, J., Williams, J., \& Tong, K. (2016). Student satisfaction and perceptions of quality: testing the linkages for PhD students. Higher Education Research \& Development, 35(6), 1153-1166. doi: https://doi.org/1 $0.1080 / 07294360.2016 .1160873$

Combs, J. P., \& Onwuegbuzie, A. J. (2012). Relationships among Attitudes , Coping Strategies , and Achievement in Doctoral-Level Statistics Courses : A Mixed Research Study. International Journal of Doctoral Studies, 7, 249375. Recuperado de https://goo.gl/UFUkhy

Cotterall, S. (2013). More than just a brain: Emotions and the doctoral experience. Higher Education Research and Development, 32(2), 174-187. doi: https://doi.org/10.1080/07294360.2012.680017

De Kleijn, R. A. M., Meijer, P. C., Brekelmans, M., \& Pilot, A. (2015). Adaptive research supervision: exploring expert thesis supervisors' practical knowledge. Higher Education Research and Development, 34(1), 117-130. doi: https://doi.org/10.1080/07294360.2014.934331

Duke, D. C., \& Denicolo, P. M. (2017). What supervisors and universities can do to enhance doctoral student experience (and how they can help themselves). FEMS Microbiology Letters, 364(9). doi: https://doi.org/10.1093/femsle/fnx090

Durette, B., Fournier, M., \& Lafon, M. (2016). The core competencies of PhDs. Studies in Higher Education, 41(8), 13551370. https://doi.org/10.1080/03075079.2014.968540

Furr, S., \& Brown-Rice, K. (2016). Doctoral students' knowledge of educators' problems of professional competency. Training and Education in Professional Psychology, 10(4), 223-230. doi: https://doi.org/10.1037/tep0000131

Gardner, S. K., Jansujwicz, J., Hutchins, K., Cline, B., \& Levesque, V. (2012). Interdisciplinary Doctoral Student Socialization Doctoral Student Socialization. International Journal of Doctoral Studies, 7, 377-394. Recuperado de https://goo.gl/UvrRc4

Gregor, M. A., \& O'Brien, K. M. (2015). The Changing Face of Psychology. The Counseling Psychologist, 43(8), 10901113. doi: https://doi.org/10.1177/0011000015608949

Hoyne, G., Alessandrini, J., \& Fellman, M. (2016). Doctoral Education for the Future: Through the Looking Glass. En P. Blessinger \& D. Stockley (Eds.), Emerging Directions in Doctoral Education (Innovations in Higher Education Teaching and Learning (pp. 21-38). doi: https://doi.org/10.1108/S2055-364120160000006010

Jairam, D., \& Kahl, D. H. (2012). Navigating the doctoral experience: The role of social support in successful degree completion. International Journal of Doctoral Studies, 7, 311-329. doi: https://doi.org/10.28945/1700

Juniper, B., Walsh, E., Richardson, A., \& Morley, B. (2012). A new approach to evaluating the well-being of PhD research students. Assessment \& Evaluation in Higher Education, 37(5), 563-576. doi: https://doi.org/10.1080/026029 38.2011.555816

Leech, N. (2012). Educating Knowledgeable and Skilled Researchers in Doctoral Programs in Schools of Education: A New Model. International Journal of Doctoral Studies, 7, 19-37. doi: https://doi.org/10.28945/1558

Lovitts. (2008). The Transition to Independent Research: Who Makes It, Who Doesn't, and Why. The Journal of Higher Education, 79(3), 296-325. doi: https://doi.org/10.1353/jhe.0.0006

Lunenburg, F. C. (2011). Self-Efficacy in the Workplace: Implications for Motivation and Performance, International Journal of Management, Business, and Administration, 14(1). Recuperado de https://goo.gl/aybczt

McAlpine, L., \& Amundsen, C. (2009).Identity andagency:pleasures and collegiality among thechallenges of thedoctoral journey. Studies in Continuing Education, 31(2), 109-125. doi: https://doi.org/10.1080/01580370902927378

McAlpine, L., \& Lucas, L. (2011). Different places, different specialisms: similar questions of doctoral identities under construction. Teaching in Higher Education, 16(6), 695-706. doi: https://doi.org/10.1080/13562517.2011.570432

McLaughlin, C. (2003). The feeling of finding out: the role of emotions in research. Educational Action Research, 11(1), 65-78. doi: https://doi.org/10.1080/09650790300200205

Melin, G., \& Janson, K. (2006). What skills and knowledge should a PhD have? Changing preconditions for PhD education and post doc work. En U. Teichler (Ed.), The formative years of scholars (pp. 105-118). London: Portlanda. Recuperado de https://goo.gl/dAkpBG

Morrison-Saunders, A., Moore, S.A., Hughes, M., \& Newsome, D. (2010). Coming to terms with research practice: Riding 
the emotional rollercoaster of doctoral research studies. In P. Walker \& M. Thomson (Eds.), The Routledge Doctoral Supervisor's Companion: Supporting effective research in Education and the Social Sciences (pp. 206-2018). London: Routledge.

Ng, I. (2017). When [inter]personal becomes transformational: [re-]examining life course-related emotions in PhD research. Area, 49(4), 409-414. doi: https://doi.org/10.1111/area.12325

Peltonen, J., Vekkaila, J., Rautio, P., Haverinen, K., \& Pyhältö, K. (2017). Doctoral Students' Social Support Profiles and their relationship to burnout, drop-out intentions, and time to candidacy. International Journal of Doctoral Studies, 12, 157-173. doi: https://doi.org/10.28945/3792

Pyhältö, K., \& Keskinen, J. (2012). Doctoral Students' Sense of Relational Agency in Their Scholarly Communities. International Journal of Higher Education, 1(2), 136-149. doi: https://doi.org/10.5430/ijhe.v1n2p136

Real Decreto 1393/2007 de 29 de Octubre por el que se establece la ordenación de las enseñanzas universitarias oficiales. Boletín Oficial del Estado, 260, pp 44037-44048. Recuperado de https://goo.gl/875o2a

Real Decreto 99/2011, de 28 de enero, por el que se regulan las enseñanzas oficiales de Doctorado. Boletín Oficial del Estado, 35, 13909-13926. Recuperado de https://goo.gl/CKyNrS

Real Decreto 1027/2011 de 15 de Julio por el que se establece el Marco Español de Cualificaciones para la Educación Superior. Boletín Oficial del Estado, 185, 87912-87918. Recuperado de https://goo.gl/VMBxg9

Reale, E., Avramov, D., Canhial, K., Donovan, C., Flecha, R., Holm, P., ... Van Horik, R. (2017). A review of literature on evaluating the scientific, social and political impact of social sciences and humanities research. Research Evaluation (Special Issue). doi: https://doi.org/10.1093/reseval/rvx025

Spaulding, L. S., \& Rockinson-Szapkiw, A. J. (2012). Hearing their voices: Factors doctoral candidates attribute to their persistence. International Journal of Doctoral Studies, 7, 199-219. doi: https://doi.org/10.28945/1589

Villardón-Gallego, L. (Coord.) (2015). Competencias genéricas en educación superior: metodologías específicas para su desarrollo. Madrid: Narcea. Recuperado de https://goo.gl/KD55qL

Villardón-Gallego, L. \& Yániz, C. (2013). Propuesta de un Plan de Tutoría y Apoyo a Estudiantes de Doctorado. Revista de Docencia Universitaria, 11(2), 135-152. doi: https://doi.org/10.4995/redu.2013.5570

Villardón-Gallego, L. \& Yániz, C. (2014). Características Psicométricas de la Escala de Estrategias Afectivas en el Proceso de Aprendizaje (EEAA). Electronic Journal of Reseach in Educational Psychology, 12(3), 693-716. doi: http:// dx.doi.org/10.25115/ejrep.34.14095

RIDU / Revista Digital de Investigación en Docencia Universitaria / ISNN 2223-2516

(c) Los autores. Este artículo es publicado por la Revista Digital de Investigación en Docencia Universitaria del Área de Institutional Research and Effectiveness de la Dirección de Aseguramiento de la Calidad, Universidad Peruana de Ciencias Aplicadas. Este es un artículo de acceso abierto, distribuido bajo los términos de la LicenciaCreativeCommons Atribución-CompartirIgual 4.0 Internacional. ( http://creativecommons.org/licenses/by-sa/4.0/), que permite el uso no comercial, distribución y reproducción en cualquier medio, siempre que la obra original sea debidamente citada. 